\title{
ANÁLISE FAUNÍSTICA E FLUTUAÇÃO POPULACIONAL DE LEPIDÓPTEROS EM Tectona grandis L.f. (LAMIACEAE) NO MUNICÍPIO DE ROSÁRIO OESTE, ESTADO DE MATO GROSSO
}

Otávio Peres Filho', Alberto Dorval ${ }^{1}$, Luiz Mário Magalhães de Souza', Evôneo Berti Filho², Rogério Goularte Moura ${ }^{2}$

'Departamento de Engenharia Florestal, Faculdade de Engenharia Florestal-UFMT, Cuiabá. CEP 78060-900, E-mail: peres@ufmt.br, adorval@terra.com.br

${ }^{2}$ Departamento de Entomologia, Fitopatologia e Zoologia Agrícola, ESALQ/USP, Piracicaba, CEP 13.418-900, Email: eberti@carpa.ciagri.usp.br,rgmoura@gmail.com

\section{RESUMO}

Foram realizados levantamentos populacionais de lepidópteros em plantios de Tectona grandis, usando 12 armadilhas luminosas, em coletas quinzenais, no período de novembro de 2000 a dezembro de 2002, no município de Rosário Oeste, estado de Mato Grosso. Foram coletados 5.492 indivíduos, sendo as famílias Hyblaeidae, Eucleidae e Noctuidae as mais representativas. Hyblaea puera (Hyblaeidae), Miresa clarissa (Eucleidae) e Dirphia rosacordis (Saturniidae) foram as espécies mais representativas em números de espécimens coletados. A espécie-praga Hyblaea puera ocorreu como super freqüente, super dominante, super abundante e constante na análise faunística. $\mathrm{Na}$ área do plantio foi encontrado um baixo índice de diversidade demonstrado pela baixa distribuição eqüitativa entre as populações de lepidópteros. Observou-se que as espécies apresentaram picos populacionais nos meses de chuva na região.

Palavras-chave: Teca, Lepidoptera, Hyblaea.

\begin{abstract}
This paper deals with a survey of lepidopteran insects in Tectona grandis plantings, by fortnight collects using light traps, in Rosário Oeste, State of Mato Grosso, Brazil, from November 2000 to December 2002. The most representative Lepidoptera families were Hyblaeidae, Eucleidae and Noctuidae, while the most representative species were Hyblaea puera (Hyblaeidae), Miresa clarissa (Eucleidae) and Dirphia rosacordis (Saturniidae).According to the faunistic analysis, Hyblaea puera was superfrequent, superdominant, superabundant and constant. One observed a low diversity index due to the low equitative distribution among the Lepidoptera populations in the $T$. grandis planting area. The species presented population peaks during the rainy season.
\end{abstract}

Key words: Teak, Lepidoptera, Hyblaea 


\section{INTRODUÇÃO}

Tectona grandis, L.f. é uma espécie florestal cultivada na Índia, Tailândia, Burma, Camboja, Indonésia, Nova Guiné, África do Sul, Sri Lanka, Indochina, Bangadlesh, Koréia, Trinidad e Tobago, Paquistão, Venezuela, Nigéria, Sumatra e Myanmar dentre outros países (Chollet \& Samapuddhi, 1967; Zethner, 1970; Torres e Silverbog, 1972; Etuk, 1973; Bell, 1973; Kamra, 1973; Ahmad, 1987; Intachat, 1998). As áreas plantadas com esta espécie florestal encontram-se em plena expansão em várias regiões do mundo. Zethner (1973) afirmou que os plantios de $T$. grandis no Pakistão e em Bangladesch apresentaram sérios problemas com várias espécies de insetos das famílias Platypodidae e Bostrichidae que atacaram as madeiras cortadas, Pyralidae, Noctuidae e Lymantriidae que causaram sérios desfolhamentos, Cerambycidae e Buprestidae que abriram galerias nos troncos das árvores e com as larvas da família Scarabaeidae causaram sérios danos às mudas em viveiros ou nas recém plantadas no campo. Darshan et al.(1975) relataram a ocorrência na Índia de lagartas de Psalis pennatuba (Lymantriidae) alimentando-se de uma grande variedade de hospedeiros, inclusive de folhas de $T$. grandis. Dhanarajan (1976) afirmou que Endoclita gmelina (Hepialidae) é uma praga de importância em áreas de plantios de $T$. grandis na Malásia causando danos consideráveis através da abertura de galerias no tronco das árvores, podendo causar a morte das árvores. Nair (1987) observou sucessivos desfolhamentos de $T$. grandis e Trema micranta (Moraceae) pela espécie Sahyadrassus malabaricus (Hepialidae). Vaishampayan et al (1987) observaram, com auxílio de armadilhas luminosas, a migração de Hyblaea puera influenciada pelos ventos das monções.

Choldumrongkul (1988) estudaram a possibilidade do uso de armadilhas luminosas para estimar as densidades populacionais de $H$. puera. Roychoudhury et al. (1995) realizaram levantamentos de insetos associados às mudas de teca em viveiro e observaram sérios danos causados por lagartas de Spodoptera litura (Noctuidae), que também desfolharam outras espécies de plantas hospedeiras. Estudos sobre biologia, ecologia e dinâmica populacional de $H$. puera foram desenvolvidos por Tilakaratna (1995) e Baksha e Crawley (1998), respectivamente. Mathew et al. (1996) afirmaram que a espécie Alcterogystia cadambae (Cossidae) é uma importante praga de $T$. grandis em algumas regiões da Índia, causando seríssimas injúrias às cascas das árvores que servem de local de entrada para fungos que podem causar a morte da árvore. Wiwatwitaya e Decha (1996) estudaram a predação de Xyleutes ceramicus Walker (Cossidae), lepidobroca em plantios de $T$. grandis na Tailândia. Veenakumari et al. (1996) observaram na ilha Andaman, Índia, severos desfolhamentos em plantios de $T$. grandis causados pelas espécies $H$. puera (Hyblaeidae), Syllepta distinguenda (Pyraustidae), Psilogramma increta (Sphingidae) e Paliga damastesalis (Pyralidae). Singh e Misra (1990) reportaram que Pagyda salvalis (Pyralidae) como importante praga em plantios de $T$. grandis na Índia e Tailândia, onde as lagartas em suas diversas fases de desenvolvimento causam severos danos às inflorescências, frutos e sementes. Avinash et al. (1998) observaram que o esqueletizador de folhas Eutectona machaeralis (Pyralidae) e o desfolhador de teca $H$. puera, causaram severos danos em quase todas as áreas plantadas com $T$. grandis na Índia.

No Brasil, principalmente, no estado de Mato Grosso, as áreas com cultivo desta espécie florestal têm se expandido devido ao alto valor comercial de sua madeira, pela boa adaptabilidade em diferentes regiões do Estado e o seu cultivo tem-se mostrado 
promissor, devido as condições edafoclimáticas favoráveis. Estudos da lepidofauna associada a esta cultura florestal no Brasil ainda são escassos. Peres Filho et al. (2002) citaram a ocorrência de $H$. puera em plantios de $T$. grandis nos municípios de Rosário Oeste e Cáceres, no estado de Mato Grosso. Os mesmos autores também citaram a ocorrência desta espécie em outras localidades brasileiras: Chapada dos Guimarães (MT), Nobres (MT), Jataí (GO), Dourados (MS) e Rio de Janeiro (RJ). Peres Filho et al. (2006) relacionaram espécies de lepidópteros $\mathrm{e}$ de outros insetos praga associados à cultura da teca no estado de Mato Grosso tanto em viveiro como no campo.

Buscando ampliar os conhecimentos sobre os lepidópteros associados à teca, e sua importância econômica objetivou-se neste trabalho efetuar estudos qualitativo, quantitativo, faunístico e de flutuação populacional com as principais espécies coletadas.

\section{MATERIAL E MÉTODOS}

Os levantamentos foram realizados em plantios homogêneos de Tectona grandis no município de Rosário Oeste, no estado de Mato Grosso, de novembro de 2000 a dezembro de 2002. O clima da região é do tipo tropical úmido, a temperatura média mensal é de $25^{\circ} \mathrm{C}$, a precipitação pluviométrica anual média é de $2.000 \mathrm{~mm}$, concentrando-se nos meses de novembro a abril, período de chuva na região. $O$ relevo da área é ligeiramente ondulado e bem drenado e com uma altitude média de 250 metros acima do nível do mar. A vegetação original é do tipo cerrado, mata de galeria e floresta estacional semi-decidual. As coletas foram feitas com armadilhas luminosas, modelo "Luiz de Queiroz", instaladas na altura de 2 metros em relação à superfície do solo. As coletas foram realizadas quinzenalmente, sendo as armadilhas ligadas das 18:00 h às 6:00 h. Os insetos coletados foram individualizados por armadilha, data de coleta e enviados para o Laboratório de Proteção Florestal, da Universidade Federal de Mato Grosso, onde passaram por triagem, contagem e etiquetagem.

Os índices faunísticos utilizados foram os seguintes: dominância, abundância, freqüência, constância e de diversidade, calculados por meio do programa ANAFAU (MORAES et al., 2003). Neste programa os dados discrepantes são analisados através da análise gráfica de resíduo, onde os valores são classificados em uma categoria própria. Também foram realizados estudos de flutuação populacional somente com as espécies que ocorreram como super dominantes, super abundantes, super freqüentes e constantes.

\section{RESULTADOS E DISCUSSÃO}

Foram coletados 14 gêneros, 15 espécies e 5.492 indivíduos distribuídos em oito famílias. Noctuidae com três espécies $(25,08 \%)$ e Arctiidae com duas (16,67\%) foram as famílias mais representativas. A família Hyblaeidae com $90,87 \%$ dos indivíduos coletados foi a mais importante (Tabela 1). As ocorrências de espécies das famílias Noctuidae, Hyblaeidae e Sphingidae em plantios de $T$. grandis foram relatadas por Tilakaratna (1995), Baksha e Crawley (1998) e Veenakumari et al. (1996), respectivamente. 
Tabela 1. Quantidade (QT) e porcentagem (\%) dos gêneros, de espécies e de indivíduos identificados taxonomicamente e que foram coletados com armadilhas luminosas em plantios de Tectona grandis. Rosário Oeste, MT, de novembro/2000 a dezembro/2002.

\begin{tabular}{l|c|c|c|c|c|c}
\hline \multirow{2}{*}{\multicolumn{1}{c}{ Família }} & \multicolumn{2}{c|}{ Gênero } & \multicolumn{2}{c|}{ Espécie } & \multicolumn{2}{c}{ Indivíduos } \\
\cline { 2 - 7 } & QT & $\%$ & QT & $\%$ & QT & $\%$ \\
\hline Arctiidae & 2 & 16,67 & 2 & 16,67 & 41 & 0,75 \\
Limacodidae & 1 & 8,33 & 1 & 8,33 & 241 & 4,39 \\
Hyblaeidae & 1 & 8,33 & 1 & 8,33 & 4.925 & 89,68 \\
Megalopygidae & 1 & 8,33 & 1 & 8,33 & 8 & 0,15 \\
Noctuidae & 3 & 25,00 & 3 & 25,00 & 116 & 2,11 \\
Psychidae & 1 & 8,33 & 1 & 8,33 & 13 & 0,24 \\
Saturniidae & 1 & 8,33 & 1 & 8,33 & 95 & 1,73 \\
Sphingidae & 1 & 8,33 & 1 & 8,33 & 44 & 0,80 \\
Yponomeutidae & 1 & 8,33 & 1 & 8,33 & 9 & 0,16 \\
\hline Total & 12 & 100 & 12 & 100 & 5.492 & 100 \\
\hline
\end{tabular}

Tabela 2. Família, gênero, espécie e números de indivíduos coletados com armadilhas luminosas em plantios de Tectona grandis. Rosário Oeste, MT, de novembro/2000 a dezembro/2002.

\begin{tabular}{|c|c|c|}
\hline Família & Gênero/Espécie & Número de Indivíduos \\
\hline Arctiidae & Paracles sp. & 3 \\
\hline Arctiidae & Utetheisa ornatrix & 8 \\
\hline $\mathrm{NI}$ & spl & 30 \\
\hline Subtotal & & 41 \\
\hline Limacodidae & Miresa clarissa & 241 \\
\hline Subtotal & & 241 \\
\hline Hyblaeidae & Hyblaea puera & 4.925 \\
\hline Subtotal & & 4.925 \\
\hline Megalopygidae & Podalia albescens & 8 \\
\hline Subtotal & & 8 \\
\hline Noctuidae & Diphthera festiva & 18 \\
\hline Noctuidae & Spodoptera frugiperda & 86 \\
\hline Noctuidae & Ceroctena sp. & 8 \\
\hline $\mathrm{NI}$ & $\mathrm{sp} .2$ & 4 \\
\hline Subtotal & & 116 \\
\hline Psychidae & Oiketicus kirbyi & 13 \\
\hline Subtotal & & 13 \\
\hline Saturniidae & Dirphia rosacordis & 95 \\
\hline Subtotal & & 95 \\
\hline Sphingidae & Protambulyx strigilis & 6 \\
\hline $\mathrm{NI}$ & sp. 3 & 38 \\
\hline Subtotal & & 44 \\
\hline Yponomeutidae & Atteva pustulella & 9 \\
\hline Subtotal & & 9 \\
\hline Total & & 5.492 \\
\hline
\end{tabular}

Hyblaea puera (Hyblaeidae) e Miresa clarissa (Limacodidae) foram, quantitativamente, as espécies mais representativas com 4.925 e 241 indivíduos coletados, respectivamente (Tabela 2). Das demais famílias (Tabela 1) várias espécies são de ocorrências bastante comuns em plantios de Eucalyptus spp. em várias regiões do Brasil, porém esta é, provavelmente, a primeira citação destas 
espécies sendo coletadas em plantios de teca no Brasil (Tabela 2). D. rosacordis, Podalia albescens, Utetheisa ornatrix, Diphthera festiva, Spodoptera frugiperda e Oiketicus kirbyi foram citadas ocorrendo em plantios de Eucalyptus spp. por Zanuncio et al. (1992); Pereira (1994); Lübeck (1995) e Viana e Costa (2001).
$\mathrm{Na}$ análise faunística dos indivíduos coletados e identificados pelo menos até ao nível de gênero, somente as espécies $H$. puera e $M$. clarissa ocorreram como super dominantes, super abundantes, super freqüentes e constantes. As demais espécies e gêneros ocorreram como não dominantes, dispersas, pouco freqüentes, acessórias ou acidentais (Tabela 4).

Tabela 3. Índices faunísticos de dominância (D), abundância $(A)$, freqüência $(F \%)$ e de constância (C) no município de Rosário Oeste, MT, de novembro/2000 a dezembro/ 2002.

\begin{tabular}{|c|c|c|c|c|}
\hline Família/Espécie & Dominância & Abundância & Frequiência (\%) & Constância \\
\hline Atteva pustulella & nd & $\mathrm{c}$ & f & $\mathrm{z}$ \\
\hline Ceroctena sp. & nd & d & pf & $z$ \\
\hline Dirphia rosacordis & d & $\mathrm{ma}$ & $\mathrm{mf}$ & $\mathrm{w}$ \\
\hline Hyblaea puera & sd & sa & sf & $\mathrm{w}$ \\
\hline Miresa clarissa & sd & sa & sf & w \\
\hline Diphthera festiva & nd & $\mathrm{c}$ & $\mathrm{f}$ & $\mathrm{y}$ \\
\hline Oiketicus kirbyi & nd & c & f & $\mathrm{y}$ \\
\hline Paracles sp. & nd & $\mathrm{d}$ & $\mathrm{pf}$ & $\mathrm{z}$ \\
\hline Podalia albescens & nd & d & $\mathrm{pf}$ & $\mathrm{z}$ \\
\hline Protambulyx strigilis & nd & $\mathrm{d}$ & pf & y \\
\hline Spodoptera frugiperda & d & $\mathrm{ma}$ & $\mathrm{mf}$ & $\mathrm{w}$ \\
\hline Utetheisa ornatrix & nd & d & pf & $\mathrm{y}$ \\
\hline
\end{tabular}

Dominância $=(\mathrm{nd})$ não dominante, $(\mathrm{d})$ dominante, $(\mathrm{sd})$ super dominante; Abundância $=(\mathrm{d})$ dispersa, $(\mathrm{c})$ comum, (ma) muito abundante, (sa) super abundante; Freqüência $(\%)=(\mathrm{pf}$ ) pouco freqüiente, (f) freqüente, (mf) muito freqüiente, (sf) super freqüente; Constância= (w) constantes, (y) acessória, (z) acidental.

Todos os índices mostraram um ambiente com uma baixa diversidade de famílias e de espécies, com a predominância populacional de $H$. puera e isto ficou evidenciado na baixa similaridade entre as populações dos lepidópteros coletados no local e que foi confirmado pelo inexpressivo índice de eqüitatibilidade de 0,1939. Quando um ecossistema é heterogêneo em quantidades de habitantes, geralmente ocorre maior quantidade de espécies, com baixas densidades populacionais, resultando em alta diversidade. Entretanto, quanto mais homogêneo for o ecossistema, normalmente ocorrerá menor quantidade de espécies, mas cada uma delas, com grandes densidades populacionais e afetando decisivamente na diversidade do local (Dajóz, 1974). 
Tabela 4. Índice de diversidade da área com plantios de Tectona grandis no município de Rosário Oeste, MT, de novembro/2000 a dezembro/ 2002.

\begin{tabular}{c|c|c}
\hline Índice de Diversidade (Shannon-Weaner) & Índice de Diversidade (Margalef) & Índice de Uniformidade ou de Eqüitabilidade \\
\hline $\mathrm{H}=0,5252$ & $\alpha=1,6256$ & $\mathrm{E}=0,1939$ \\
\hline
\end{tabular}

\section{Flutuação Populacional}

H. puera apresentou pico populacional em outubro/2001 (Figura 1) e em novembro/2002, coincidindo com o período de chuva na região da pesquisa. Nos demais meses, quando esteve presente nas áreas de $T$. grandis, esta espécie ocorreu com baixas densidades populacionais, pois talvez maior quantidade de indivíduos estivessem em hospedeiros nativos próximos às áreas plantadas. Nair et al. (1985) observaram em algumas regiões da Índia, em plantios de $T$. grandis, grandes densidades populacionais de $H$. puera causando severos desfolhamentos, com os principais picos populacionais ocorrendo nos meses de abril, junho e no período de agosto a outubro diferindo dos resultados obtidos no estudo da flutuação populacional dessa espécie no município de Rosário Oeste, MT. Os resultados obtidos por Vaishampayan et al. (1987) sobre a ocorrência de $H$. puera em diversas regiões da Índia confirmaram que a densidade populacional desta espécie é totalmente dependente do clima de monções e que seus principais picos populacionais ocorrem no período de maio a outubro e decrescendo a densidades mínimas em janeiro.

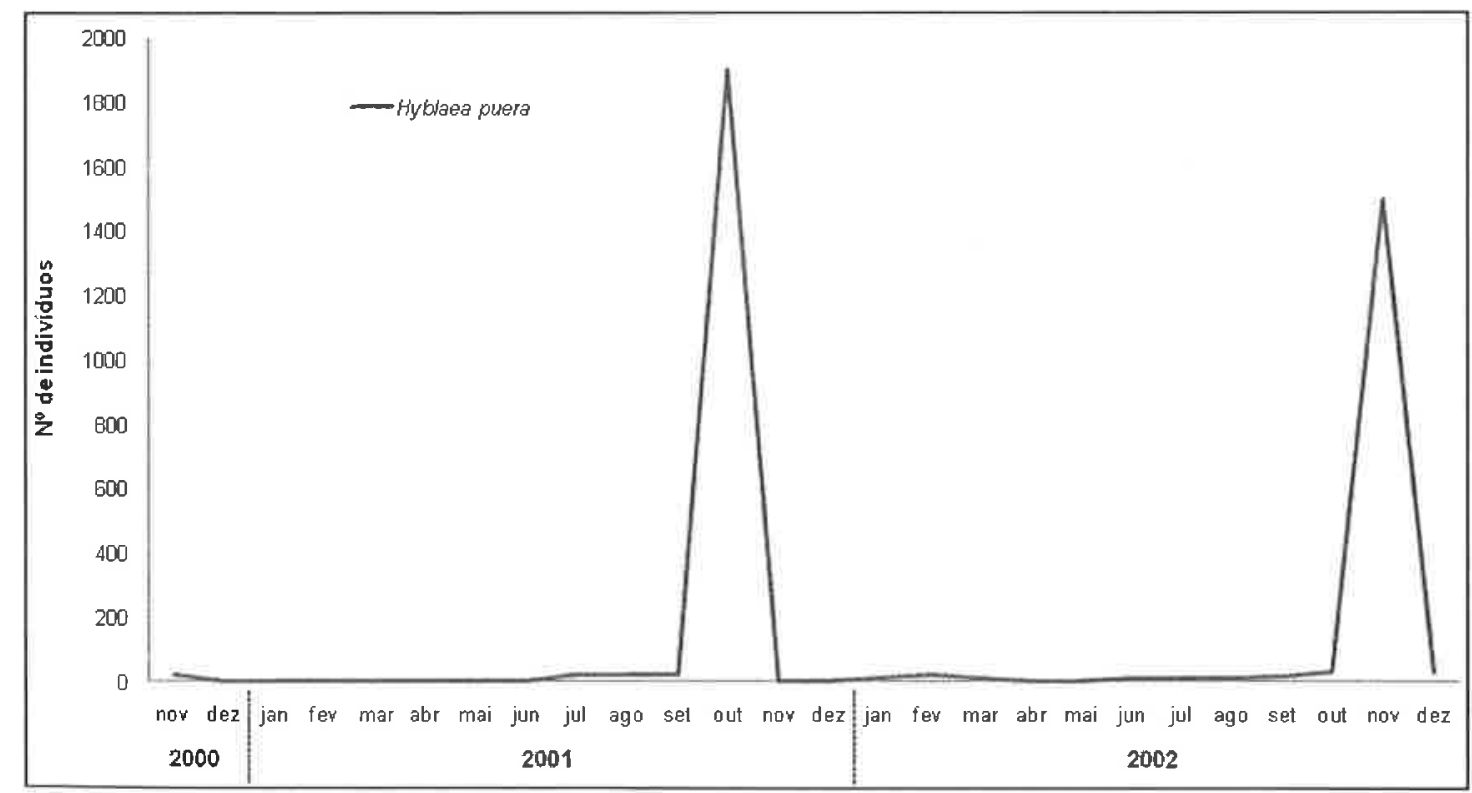

Figura 1. Flutuação populacional de Hyblaea puera (Hyblaeidae) em plantio de Tectona grandis Lamiaceae) no município de Rosário Oeste, MT, de novembro/2000 a dezembro/2002.

A espécie $M$. clarissa ocorreu associada aos surtos populacionais de $H$. puera, porém, sempre com baixas densidades populacionais durante todo o período de coletas. Essa espécie apresentou pico populacional em agosto/2001 e elevação nas quantidades de indivíduos coletados em junho, julho e de outubro a 
dezembro (Figura 2). Nos meses de fevereiro, abril e outubro/2002 ocorreu com picos populacionais e elevações nas quantidades de indivíduos coletados nos meses de julho e setembro (Figura 2). $\mathrm{Na}$ literatura especializada não foi constatada nenhuma referência sobre a ocorrência desta espécie associada a plantios de $T$. grandis.

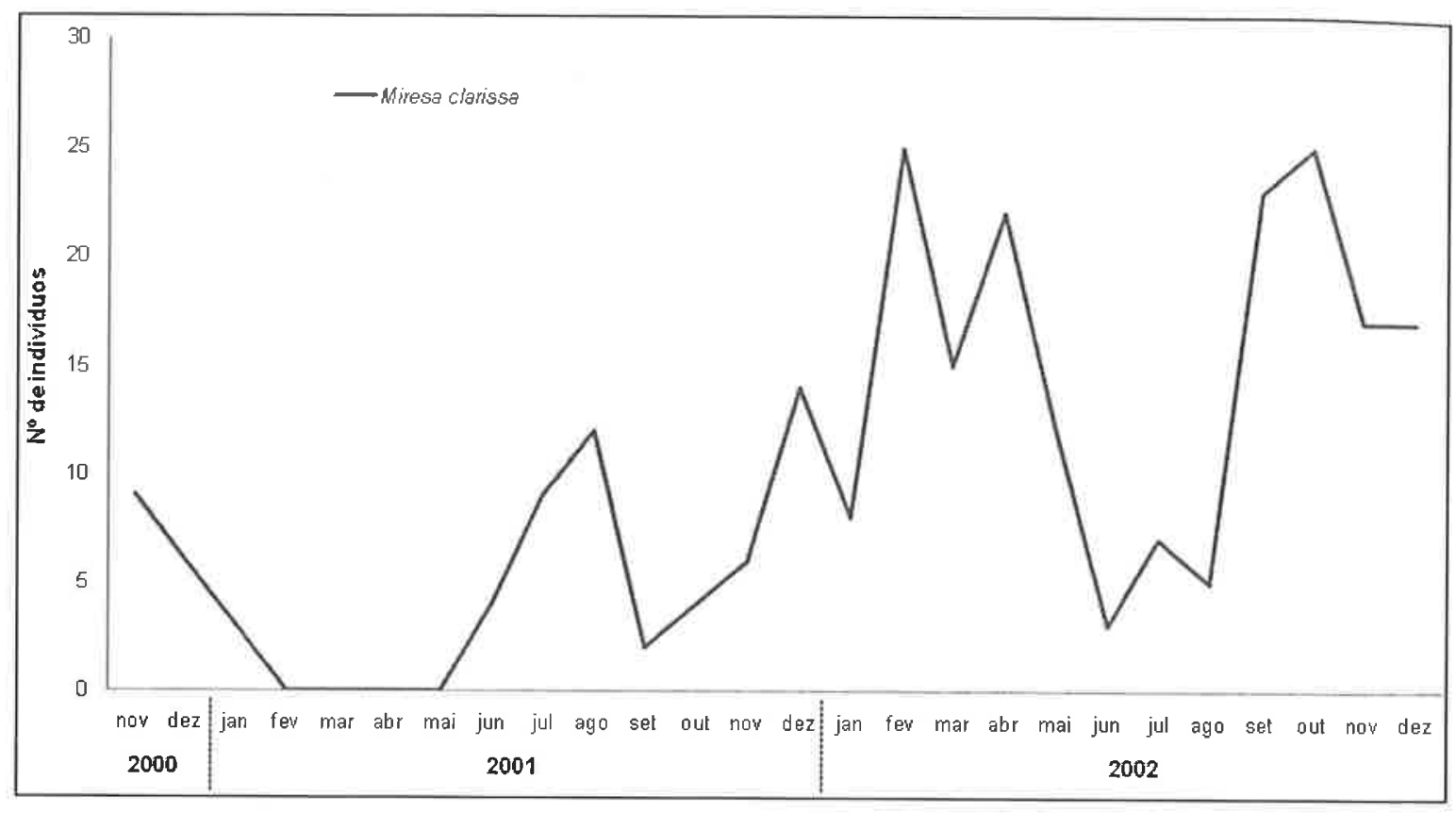

Figura 2. Flutuação populacional de Miresa clarissa (Limacodidae) em plantio de Tectona grandis (Lamiaceae) no município de Rosário Oeste, MT, de novembro/2000 a dezembro/2002.

\section{CONCLUSÕES}

Hyblaea puera é quantitativamente a espécie mais representativa dentre todas as espécies de lepidópteros coletados. Miresa clarissa e Dirphia rosacordis encontram-se em fase de adaptação aos plantios de teca em Mato Grosso. Hyblaea puera e Miresa clarissa apresentam altos picos populacionais no período das chuvas; A baixa equitatividade entre as espécies coletadas, evidenciou o potencial de ocorrência de surtos de insetos praga na área amostrada.

\section{AGRADECIMENTO:}

Ao técnico Manoel Lauro da Silva, do Laboratório de Proteção Florestal, da Faculdade de Engenharia Florestal/UFMT, pela inestimável ajuda na realização deste trabalho.

\section{REFERÊNCIAS BIBLIOGRÁFICAS}

AVINASH, J. ; ROYCHOUDHURY, N.; SANDEEP, S.; ALKA, B.; PANT, N.C.; JAIN, A.; SHARMA, S.; BHARGAVA, A. 1998. Host plant resistance to insect-pests in teak (Tectona grandis L.F.) with reference to biochemical parameters. Indian Journal of Forestry, v.21, n.4, p. 285289.

BAKSHA, M.W.; CRAWLEY, M.J. 1998. Effect of defoliation on the growth of teak. Journal of the Tropical Forest Science, v.10, n. 3, p. 312-317.

BELL, TIW. 1973. Erosion in the Trinidad teak plantations. Commonwealth 
Forestry Review. v. 52, n.3, p. 223233.

CHOLLET, A.; SAMAPUDDHI, K. 1967. Teak sub-commission. Fourth Session (Rome, 17 octubre 1967). Provisional Agenda. FAO Reports., v. 67, n. 1-8 45p.

DAJOZ, R. 1974. Tratado de ecologia. Madrid, Mundi, 487p.

DARSHAN, S.; RAMZAN, M.; SINGH, D. 1975. Brinjal as a new host of yellow hairy caterpillar, Psalis pennatula (F.) (Lymatriidae;Lepidoptera). Current Science. v.44, n. 5, p.176.

DHANARAJA, G. 1976. Some observations on the teak collar ringe borer Endoclita gmelina Tindale (Lepidoptera; Hepialidae) in north western Malaysia. Malaysian Forester, v.39, n.4, p.214223.

ETUK, E. L 1973. A preliminary survey of the weeds in teak plantations in the Western State of Nigeria. Obeche, v. 8-9, p. 37-48.

INTACHAT, J. 1998. The identity of a Malaysian teak skeletonizer, Paliga damastesalis Walker (Lepidoptera; Pyralidae). Journal of Tropical Forest Science. v.10, n.4, p. 561-563.

HUTACHARERN, C.; CHOLDUMRONGKUL, S. 1988. Possibility of using light traps to estimate the population of the teak defoliators. Thai Journal of Forestry (Thailand). v.7, n.1, p.28-36.

KAMRA, S.K. 1973. Forestry seed problems of some developing countries in Asia. Sri Lanka Forester, v. 11, n. 1-2, p. 512.

LÜBECK, G.M.; OLIVEIRA, J.V.; ALMEIDA, R.P. 1995. Análise faunística de lepidópteros coletados em duas comunidades agrícolas na zona da mata, norte de Pernambuco. An. Soc. Entomol. Brasil, v.24, n.2, 353-370.

MATHEW, G.; RUGMINI, P.; NAIR, K.S.S.; SHARMA, J.K.; VARMA,
R.V. 1996. Impact of the borer Alcterogystia cadambae (Moore)(Lepidoptera;Cossidae) in forest plantations of the teak in Kerala, Índia. In: IUFRO. IMPACTO OF DISEASES AND INSECT PESTS IN TROPICAL FORESTS. SYMPOSIUM. 1996, Pecchi, India, p.304-310.

MARGALEF, R. 1974. Ecologia. Ediciones Omega, Barcelona, Espanha. 600p.

MORAES, R.C.B. 2003. Software para análise faunística. IN: SIMPÓSIO DE CONTROLE BIOLÓGICO, 8, São Paulo, Resumos, Piracicaba: ESALQ/USP, p.195..

NAIR, K. S. S.; SUDHEENDRAKUMAR, V.V.; VARMA, R.V.; CHACKO, K.C. 1985. Studies on the seasonal incidence of defoliators and the effect of defoliation on volume incremento $f$ teak.. Forest Research Institute, n. 30, $78 \mathrm{p}$.

NAIR, K.S.S. 1987. Control of the sapling borer, Sahyadrassus malabaricus (Lep.; Hepialidae) in forest plantations. Entomon, v.12, n.2, p.137-139.

PEREIRA, J.M.M.; ZANUNCIO, J.C.; SCHOEREDER, J.H.; GASPERAZZO, W.L. 1994.Índices faunísticos e flutuação populacional de lepidópteros daninhos ao eucalipto na região de Montes Claros, Minas Gerais. An. Soc. Entomol. Brasil, v.23, n.2, p. 324-334.

PERES FILHO, O.; DORVAL, A.; BERTI FILHO, E. 2002. Ocorrência de Hyblaea puera (Cramer, 1777) (Lepidoptera: Hyblaeidae) em teca no Brasil. Bragantia, Campinas, v. 61, $\mathrm{n}$. 1, p. 59-60.

PERES FILHO, O.; DORVAL, A.; BERTI FILHO, E. 2006. A entomofauna associada à Teca, Tectona grandis L.f. (Verbenaceae) no Estado de Mato Grosso. Piracicaba, SP: IPEF, Elbergráfica Artes Gráficas Ltda., 58 p. 
ROYCHOUDHURRY, N.; SHAMILA, K.; JOSHI, K.C.; KALIA, S. 1995. Pest status and larval feeding preference of Spodoptera litura (Fabricius) Boursin (Lepidoptera: Noctuidae) on teak. Focus on teak. Indian Forester, v. 121, n.6, p. 581-583.

SINGH, P.; MISRA, R. M. 1990. External morphology, bionomics and natural enemy complex of Pagyda salvalis Walker (Lepidoptera; Pyralidae) the inflorescence feeder and fruit borer of teak. Indian Forester, v.116, v.9, p. 742-747.

TILAKARATANA, D. 1995. Life history of the defoliator, Hyblaea puera. Sri Lanka Forester. v. 22, n. 1-2, p. 2528.

TORRES, L.; SILVERBORG, S.B. 1972. Study on the natural durability of teak by means of accelerated soli/block tests in the National Forest Products Laboratory, Mérida, Venezuela. Boletin do Instituto Forestal Latino Americano, n. 41-42, p. 63-70.

VAISHAMPAYAN， S.M.;VERMA， R.; BHOWMICK. 1987. Possible migration of teak-defoliator, Hyblaea puera Cramer (Lepidoptera: Hyblaeidae) in relation to the movement of the south-west monsoon as indicated by light trap catches. Indian Journal of Agricultural Sciences. . v. 57, n.1, p. 41-46.
VEENAKUMARI, K.; PRASHANTH, M.; MOHANRAJ, P. 1996. Folivorus insects damaging teak Tectona grandis L. (Lamiaceae) in the Andaman Islands, Bay of Bengal, Indian Ocean. Journal of Entomological Research, v. 20, n.2, p. 177-178.

VIANA, T.M.B.; COSTA, E.C. 2001. Lepidópteros associados a duas comunidades florestais em Itaara, RS. Ciência Florestal, v.11, n.1, p.67-80.

WIWATWITAYA, D.; DECHA, W. 1996. Predador ants of the teak bechole borer, Xyleutes ceramicus Walker (Lepidoptera; Cossidae). Kasetsart Journal Natural Sciences. v.30, n.3 p. 330-335.

ZANUNCIO, C. J.; FAGUNDES, M.; ZANUNCIO, T.V.; MEDEIROS, A. G. B. 1992. Principais lepidópteros, pragas primárias e secundárias, de Eucalyptus grandis na região de Guanhães, Minas Gerais, durante o período de junho de 1989 a maio de 1990 . Científica , São Paulo, v.20, n.1, p. 145-155.

ZETHNER, O. 1970. Defoliations of Teak by Hyblaea puera Cr. In East Pakistan. Forest Dale News, v. 2, n. 4, p. 45-49.

ZETHNER, O. 1973. Entomological problems in forests in the Indian subcontinent with examples from Pakistan and Bangladesh. Entomologiske Meddelelser. v. 41, n.3, p. 129-14 